\title{
Development and application of an automatic measurement method for nozzle orifice diameter and length*
}

\author{
Zhi-long $\mathrm{LI}^{1,2}$, Zhi-jun $\mathrm{WU}^{\dagger \ddagger 2}$, Ya GAO ${ }^{2}$, Wei-di HUANG ${ }^{2}$, Hui-feng $\mathrm{GONG}^{2}$, Lin ZHANG ${ }^{2}$, Li-guang $\mathrm{LI}^{1}$ \\ ( ${ }^{1}$ School of Mechanical Engineering, Tongji University, Shanghai 200092, China) \\ ( ${ }^{2}$ School of Automotive Studies, Tongji University, Shanghai 200092, China) \\ †E-mail: zjwu@tongji.edu.cn
}

Received Apr. 29, 2014; Revision accepted Aug. 18, 2014; Crosschecked Dec. 8, 2014

\begin{abstract}
Diesel sprays are important to engine combustion and emission formation processes, however, their behaviors are difficult to predict as they are very sensitive to the internal geometries of the nozzle. Based on synchrotron radiation X-ray tomography, a novel method is presented for measuring automatically the orifice diameter and length of fuel nozzles. According to this method, a clear definition of orifice inlet and outlet is given, and the diameters along the orifice from inlet to outlet as well as the orifice length can be measured. Measurements of a single-hole nozzle and an eight-hole nozzle have been performed accordingly. The results show that this method can automatically measure the orifice diameters from outlet to inlet along the whole orifice axis with relatively high precision, regardless of whether it is a single-hole or a multi-hole nozzle. The profile of the diameters obtained shows the differences between the nominal dimensions and the actual ones, which gives a more precise feedback for nozzle manufacture, and provides a new basis for precisely studying the impacts of internal geometries on spray behavior.
\end{abstract}

Key words: Diesel nozzle, Orifice diameter, Orifice length, X-ray tomography doi: $10.1631 /$ jzus.A1400118

Document code: A

CLC number: TH71

\section{Introduction}

Fuel injection in the combustion chamber has a significant influence on the fuel spatial distribution, evaporation, ignition, combustion, and therefore the formation of pollutant emissions in diesel engines (Reitz, 1978). For this reason, diesel sprays have been studied for many years, covering aspects from the spray's penetration and cone angle (Araneo et al.,

\footnotetext{
Corresponding author

* Project supported by the National Natural Science Foundation of China (Nos. 91441125, 51106113, and 51376139), the New Century Excellent Talents (No. NCET-10-0605), the Specialized Research Fund for the Doctoral Program of Higher Education of China (No. 20120072110015), the China Postdoctoral Science Foundation (No. 2013M531209), and the Fundamental Research Funds for the Central Universities, China

(16) ORCID: Zhi-long LI, http://orcid.org/0000-0002-0167-4037; Zhijun WU, http://orcid.org/0000-0002-5430-2667

(C) Zhejiang University and Springer-Verlag Berlin Heidelberg 2015
}

1999; Gao et al., 2009) to the velocity of droplets and their diameter distributions in the spray (Wu et al., 2006; Dong et al., 2013). However, most of these studies, including experiments and simulations, have limitations in exactly predicting spray behavior, because of the impacts of orifice inner geometries and dimensions, which have been proven to be key influences of spray behavior (Ohrn et al., 1991; Payri et $a l ., 2008)$, and they cannot be replaced by nominal ones (Huang et al., 2013a; 2013b). To optimize fuel sprays, the nozzle orifice is designed to be very tiny (of the order of $100 \mu \mathrm{m}$ ), but the manufacturing of such orifices exactly to the nominal dimensions is hard. The differences between the actual dimensions and the nominal ones have hindered researchers in comparing and analyzing spray behavior performed at different facilities.

To overcome this limitation, several strategies have been developed for measuring the geometries of 
diesel nozzle orifices (Kastengren et al., 2012). These methods can be classified into four groups: optical microscopy of the nozzle exit (Diver et al., 2004), a micro probing test (Kao and Shih, 2007; Peiner et al., 2009), the silicone modeling method (Macian et al., 2003), and the synchrotron radiation X-ray imaging method (Fezzaa et al., 2005). These methods normally have problems of either low accuracy or high cost. For example, the studies of the probing method are still in the proof-of-concept stage, and the shrink of silicone models during solidifying causes unpredictable problems when measured by scanning electron microscopy (SEM). Fezzaa et al. (2005) used a synchrotron based phase-contrast X-ray imaging approach to observe the internal structure of nozzle tips. However, the line-in-sight images will cause the overlap of orifices while dealing with multi-hole nozzles.

In recent years, the X-ray computerized tomography (CT) scan has been applied to reconstruct 3D digital models of nozzle tips for the measurements of their inner structures with resolution of $3.7 \mu \mathrm{m}$ (Wu et al., 2012), based on which some predictions of spray macro scope behaviors have been presented with higher accuracy (Gao et al., 2013; Huang et al., 2013a). However, in normal practice, only the diameters at the orifice inlet and outlet are measured and applied to predict spray behaviors, and the variability of the diameters along the orifice axis have been ignored due to the lack of suitable approaches. And most of all, the definition of orifice inlet, outlet, and length are ambiguous. Although they are crucial for the simulation of spray behaviors, the determination of these key structures is still dependent on the measurement operators, as all these methods need a manual process to measure the dimensions.

In this study a novel method is presented to acquire more precise internal geometries automatically. Based on 3D digital models of the nozzle tip, a clear definition of orifice inlet and outlet is given, and the diameter profile along the whole orifice axis and the length of the orifice can be obtained. This geometry information provides a precise feedback for nozzle manufacturing, and also it can provide the most precise geometrical boundary conditions for the modeling of fuel internal flow and primary atomization of diesel sprays.

\section{Orifice 3D digital models}

To obtain 3D digital models of nozzle tips, synchrotron radiation X-rays were applied. Compared to the normal light source, these high energy X-ray beams have excellent performance: high intensity and high stability. In this study, the internal structures of diesel nozzle tips were revealed on the X-ray imaging and biomedical applications beam line (BL13W1, Shanghai Synchrotron Radiation Facility, China). The experimental setup is sketched in Fig. 1a. The diesel nozzle is held on the specimen rotating platform, and the X-ray penetrates its tip and is irradiated on the scintillator crystal to convert the X-ray to visible light, to be recorded by a charge coupled device (CCD) camera. The pixel size of the camera is $7.4 \mu \mathrm{m} \times 7.4 \mu \mathrm{m}$ corresponding to an actual spatial resolution of $3.7 \mu \mathrm{m} \times 3.7 \mu \mathrm{m}$ with a $2 \times$ objective lens. The exposure time of each single frame was set to $8 \mathrm{~s}$, and the photon energy of X-ray was tuned to $50 \mathrm{keV}$. Absorption images were taken every $0.25^{\circ}$ with the nozzle rotating and totally 720 absorption images were captured during the $\mathrm{CT}$ scanning process.

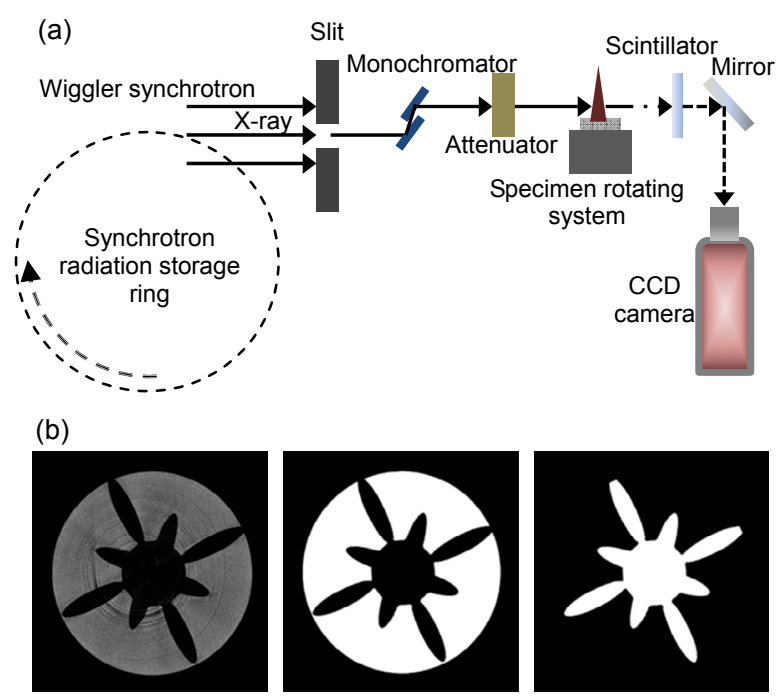

Fig. 1 Procedure of recording the inner structures of nozzle tip by high energy $\mathrm{X}$-rays (a) and the original slice (left), binary slice (middle), and reverse slice (right) (b)

In addition, a reconstruction process generated these absorption images as slices, by which a $3 \mathrm{D}$ digital model of the diesel nozzles was constructed (Fig. 1b). However, the original slices might have the defect of low signal to noise ratio, and thus they were 
converted to binary images and reverse images (Fig. 1b). With the nozzle tip model stacked by orifice binary images, the geometries can be observed and measured effectively. The orifices of an eight-hole nozzle and a single-hole nozzle are displayed in Fig. 2.

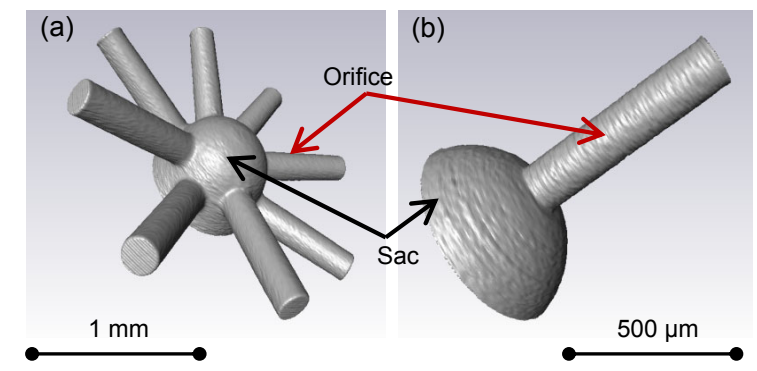

Fig. 2 Typical 3D-digital models reconstructed from synchrotron $X$-ray tomography: (a) eight-hole nozzle; (b) single-hole nozzle

\section{Measurement procedure}

To increase the nozzle discharge coefficient, modern diesel nozzles are normally processed by electrical discharge machining (EDM) (Diver et al., 2004) to form tapered cone shaped orifices, and they have also undergone hydro-grinding to smooth the interior contours of the nozzle. Therefore, the orifice inlet diameter is a little larger than that of the outlet. The $K$-factor of an orifice describes this convergence of the nozzle orifice between inlet $\left(d_{\mathrm{i}}\right)$ and outlet $\left(d_{\mathrm{o}}\right)$ diameters (Macian et al., 2003), and is defined as $K=\left(d_{\mathrm{i}}-d_{\mathrm{o}}\right) /(10 \mu \mathrm{m})$.

However, as the 3D digital models obtained from the synchrotron X-ray tomography are just binary arrays stored in computers, the value 1 or 0 at each point indicates only that it is inside or outside the orifice, so the acquisition of orifice dimensions according to such a set of numbers needs a series of further processes.

\subsection{Single-hole nozzle}

According to Apollonius (1896)'s treatise on conic sections of Apollonius, when a plane intersects with a cone, the intersection image can be a circle or an ellipse depending on their intersection position. If the intersection plane is perpendicular to the symmetry axis of the cone, as Plane 1 in Fig. 3, the obtained intersection image will be a circle. This theory has laid a foundation for the automatic measurement of orifice geometry dimensions. The basic principle of the testing idea is listed in the following steps:

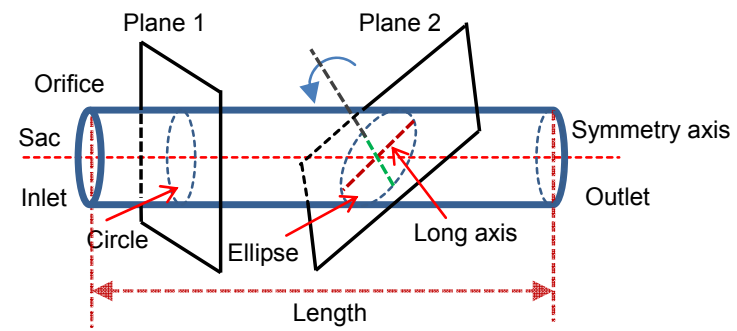

Fig. 3 Sketch of intersection images between planes and nozzle orifice

Firstly, choose an arbitrary plane that intersects with the orifice, as Plane 1 or 2 in Fig. 3. If the obtained intersection image is a circle, this selected plane must be perpendicular to the cone axis, otherwise the intersection image will be an ellipse. Then the best fitting ellipse according to the intersection image will be simulated by the least square method. The directions of the short and long axes of this ellipse can be determined in this 3D model. By rotating the intersection plane around the short axis, there will always be a specific angle which makes this plane perpendicular to the orifice axis. In Fig. 3, Plane 2 shows the rotating process around the short axis.

Secondly, this obtained circle will be used to determine the orifice axis, which should pass through the center of the circle and be perpendicular to the circle plane. Then using a few planes that are perpendicular to the axis to cut the orifice along its axis, the roundness of every circle in their intersection images can be tested to check the validation of the orifice axis. If it fails, the first step should be carried out again to find a valid one.

Thirdly, the planes perpendicular to the axis can be used to cut the orifice pixel by pixel, to obtain the intersection images along the orifice; then the whole diameters can be calculated accordingly. Normally the areas of the intersection images increase linearly and slowly from outlet to inlet. Sudden area changes only happen at two locations. One takes place at the inlet, where the orifice connects to the sac, so the intersection areas will increase significantly when the sectioning plane passes from the inlet to the sac. The other one is located at the outlet, at the end of the orifice, so the intersection areas will decrease 
suddenly when the sectioning plane passes by the outlet. Thus, the two locations where the intersection areas change largely and deviate from linear growth on their two sides will be considered as the inlet and outlet, which will be discussed in detail in the following sections. The length between the inlet and outlet should be the orifice length (Fig. 3).

However, the previous steps were proposed based on the ideal nozzle 3D models, whereas the real models of the nozzle orifices cannot be a perfect tapered cone shape. So among these steps there are some issues that should be dealt with, such as the judgment of intersection image roundness, which is crucial for the determination of the orifice axis. However, due to the defects in manufacture, it is almost impossible to identify an absolute circle when rotating the plane. In regular determinations, roundness has been used in the mechanical and measurement area for many years (Bryan, 1982), but those determining methods usually compare a particular circle (such as circumscribed circle or inscribed circle) with the nominal one to calculate the roundness. They are not suitable for the testing of intersection images as it is hard to determine a local nominal circle of the orifice when the plane is arbitrarily settled. In this study, an ellipse fitting method was applied to test the roundness of the intersection images. According to this method an ellipse is simulated to fit the intersection image (Fitzgibbon et al., 1999). After obtaining the ellipse center, the disparity between the short and long axis can provide a criterion for the judgment of roundness, which can be demonstrated as $\delta=(L-S) / L$, where $S$ and $L$ are the length of short axis and long axis, respectively. The smaller $\delta$ is, the closer the ellipse is to a circle.

During the experiment, it was found that when $\delta<0.01$, the ellipse will be very close to an absolute circle. So in this study the ellipse will be treated as a circle for subsequent processing when $\delta<0.01$. Then a few planes parallel to this circle will be arbitrarily chosen to check the validation of the axis, if these intersection images can meet the roundness judgment, then the obtained orifice axis is valid, otherwise it should be determined again.

\subsection{Multi-hole nozzles}

When dealing with multi-hole nozzles, their orifices are located around the nozzle tip, and they are very close to each other (Fig. 4b), so the previous steps are not practicable. When using a plane to cut one orifice, it would intersect with other adjacent orifices. Thus, the 3D models of nozzle tip should be pre-processed. As the testing process is independent, the measurement of each orifice can be done individually. By assigning the starting angle $\left(R_{1}\right)$ and ending angle $\left(R_{2}\right)$ shown in Fig. 4b manually, one orifice can be separated from the whole nozzle tip. Thus, the orifice shown in Fig. 4a can be treated as a single-hole nozzle to obtain the internal geometries directly.

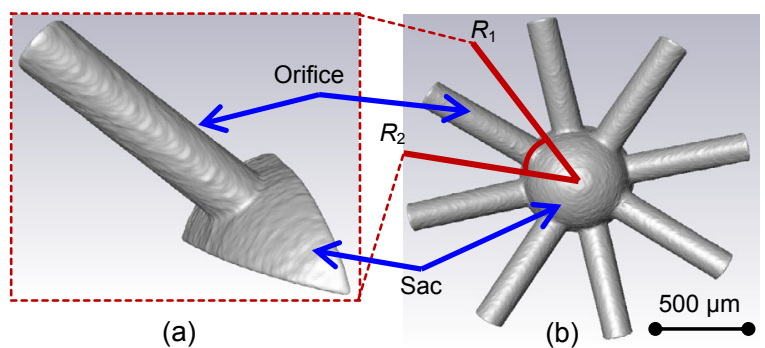

Fig. 4 Sketch of extraction of one orifice from a multi-hole nozzle model

\section{Measurement and analysis}

Two different nozzles were tested in this contribution, one is a special designed single-hole nozzle and the other is an industrial eight-hole nozzle. Their orifice structures can be found in Fig. 2. The orifices of the two nozzles are designed to be tapered cone shapes with a $K$-factor of 2 ; the nominal outlet diameter is $160 \mu \mathrm{m}$.

\subsection{Measurement and analysis of single-hole nozzle}

The intersection images along the orifice axis are extracted at each pixel corresponding to an actual spatial interval of $3.7 \mu \mathrm{m}$, as $S_{1}, S_{2}$, and $S_{3}$ shown in Fig. 5. Based on these intersection images, the diameters can be measured according to the circle areas. However, this method, which only counts the pixel numbers of each circle, is not sensitive to the shape of the intersection image. So the selection of slices near the orifice inlet and outlet needs careful treatment.

The diameter distribution result is shown in Fig. 6. From the diameter profile, it can be found that for a single-hole nozzle the outlet and inlet of the 
orifice are very easily identified. As the obtained value belonging to the orifice can fit a very obvious straight line, at the left side, the break of this trend determines the outlet. As the orifice inlet is the separation of the orifice and the sac, based on the inlet definition, the inlet can be identified easily as there is a sudden increase in diameter when the sectioning plane moves from the orifice to the sac (Fig. 6). The distance between the outlet and inlet is the length of the orifice. As the thickness of every intersection image is $3.7 \mu \mathrm{m}$, and in total 188 images are obtained, the length of the orifice is $695.6 \mu \mathrm{m}$.

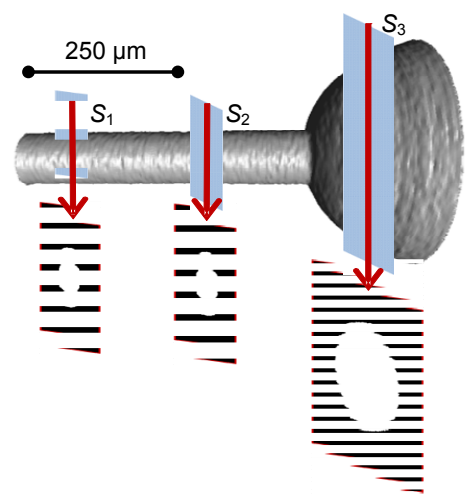

Fig. 5 Typical intersection images along the single-hole nozzle orifice

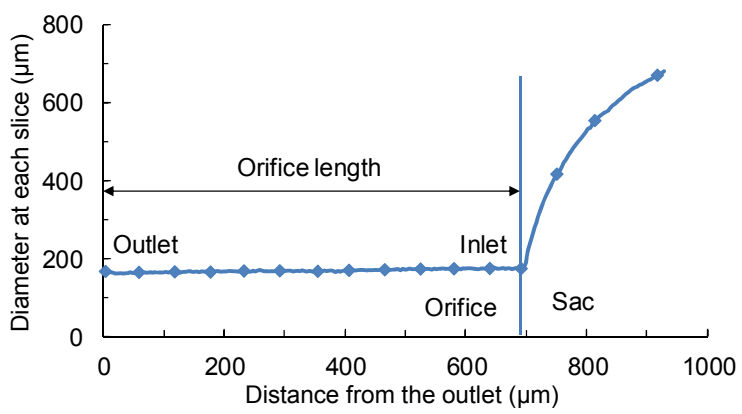

Fig. 6 Diameters along the single-hole nozzle orifice

The diameter profile along the orifice was fitted to a straight line, and the formula of this line is described as $D(x)=0.0179 x+162.44$, where $x$ is the distance from the outlet in $\mu \mathrm{m}$. The linearly dependent coefficient $R^{2}$ of this fitting is 0.91 , which is quite close to 1 , indicating that the linearity of the orifice diameter from outlet to inlet is quite good. The outlet diameter, where $x=0$, is $D(0)=162.44 \mu \mathrm{m}$, which is very close to its nominal value of $160 \mu \mathrm{m}$. The inlet diameter, where $x$ is equal to the orifice length, is $D(695.6)=174.9 \mu \mathrm{m}$, so the inlet diameter is not a good fit to the design value.
The measured $K$-factor of this orifice is around 1.3, much smaller than the expected nominal value of 2 .

\subsection{Measurement and analysis of the multi-hole nozzle}

For multi-hole nozzles, the joint face of the orifice and the sac is very different from that in the single-hole nozzle. The intersection slices of the outlet area are shown in Fig. 7 labeled with $O_{1}-O_{6}$, and it can be seen that there is no sudden ending as in the single-hole nozzle. Although the intersection images in these slices are partial circles, it does not mean that the axis of this orifice is a wrong selection. This is caused by a little abnormality between the orifice axis and the outside of the sac wall. So it is difficult to determine the orifice outlet. However, in this study the $O_{6}$ slice with a full circle has been chosen to be the outlet according to the outlet definition, as when the fuel is flowing through this place, it will be influenced directly by the ambient air.

The inlet determination encountered a similar situation. The intersection images of this area are described as $I_{1}-I_{4}$ slices. When the cutting plane is very close to the sac area, part of the sac will be cut and displayed on the slices, as $I_{2}$ and $I_{3}$ shows. In $I_{4}$ slice, the intersection image is not a circle any more, thus the location of $I_{3}$ was selected to be the inlet of this orifice according to the inlet definition. The length of the orifice is obtained after the outlet and inlet are determined. There are totally 169 slices from the outlet to the inlet, so the orifice length is $625.3 \mu \mathrm{m}$.

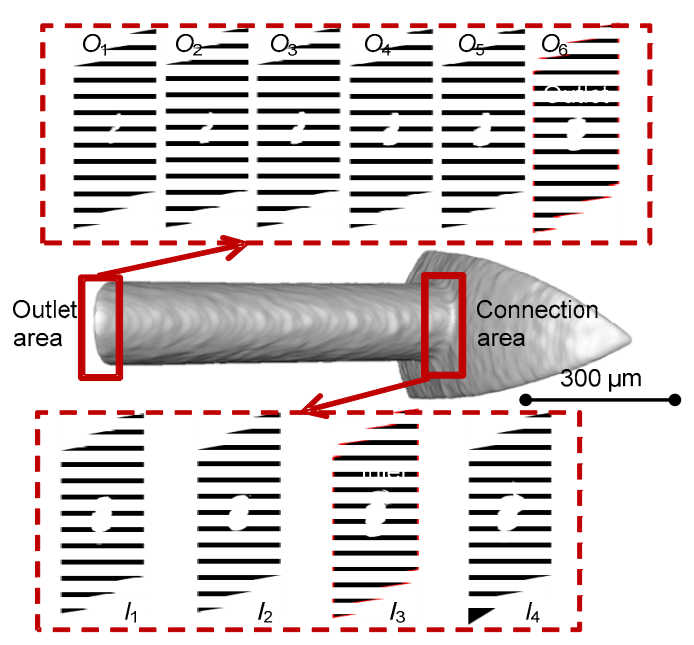

Fig. 7 Typical intersection images of multi-hole nozzle 
The diameter profile is displayed in Fig. 8, and it was also fitted to a straight line, as $D(x)=0.0280 x+$ 164.38 , and $x$ is the distance from the outlet in $\mu \mathrm{m}$ as well. The linearly dependent coefficient $R^{2}$ of this fitting is 0.98 , which is very close to 1 , so it means this orifice has a much better linearity than the singlehole nozzle. Based on this formula, the calculated outlet diameter of this orifice is $D(0)=164.4 \mu \mathrm{m}$, and the inlet diameter is $D(625.3)=181.9 \mu \mathrm{m}$. The outlet and inlet diameter are similar to the nominal values, and the $K$-factor is 1.75 , which is closer to the nominal value than in the case of the single-hole nozzle.

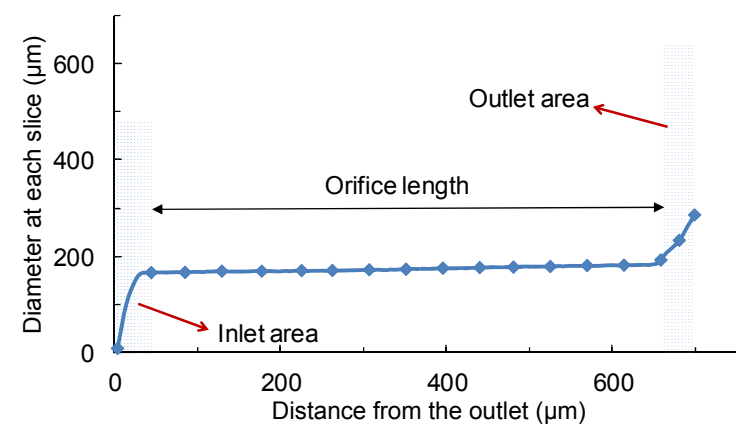

Fig. 8 Orifice diameters along the multi-hole nozzle orifice axis

\section{Conclusions}

Based on the diesel nozzle 3D digital models obtained from the synchrotron radiation X-ray tomography technology, a new orifice diameter and length measurement method was presented. According to this method, a single-hole nozzle and an eighthole nozzle have been measured and analyzed. According to the results, the following conclusions can be drawn:

1. This method is operable and according to the results, the orifice diameters and lengths of singlehole and multi-hole nozzles can be measured regardless of the resolution and the source of the digital models. So the nozzle tip digital models obtained from different X-ray CT scanners with different resolutions can also be treated by this method.

2. The pre-process method for the multi-hole nozzle is reasonable, by which the multi-hole nozzle can be treated as a single-hole nozzle to measure the diameters and lengths of its orifices.

3. Clear definitions of orifice inlet and outlet have been drawn in this paper, and they provide a practical way to identify these important structures, and lays a foundation for future measurements and studies.

4. By this method, the diameter profile along the whole orifice can be observed. The linearity of the diameter distribution from the outlet to the inlet gives a new aspect to researching the manufacturing technology of nozzle orifices, and can also be used to describe the internal geometry features of the orifice for the simulation of fuel inner flow.

\section{References}

Apollonius, 1896. Treatise on Conic Sections. Cambridge University Press, Cambridge, England.

Araneo, L., Coghe, A., Brunello, G., et al., 1999. Experimental investigation of gas density effects of diesel spray penetration and entrainment. SAE Technical Paper 1999-010525. [doi:10.4271/1999-01-0525]

Bryan, J.B., 1982. A simple method for testing measuring machines and machine tools. Part 1: principles and applications. Precision Engineering, 4(2):61-69. [doi:10. 1016/0141-6359(82)90018-6]

Diver, C., Atkinson, J., Helml, H.J., et al., 2004. Micro-EDM drilling of tapered holes for industrial applications. Journal of Materials Processing Technology, 149(1-3): 296-303. [doi:10.1016/j.jmatprotec.2003.10.064]

Dong, Q., Long, W.Q., Ishima, T., et al., 2013. Spray characteristics of V-type intersecting hole nozzles for diesel engines. Fuel, 104:500-507. [doi:10.1016/j.fuel.2012.07. 021]

Fezzaa, K., Lee, W.K., Cheong, S.K., et al., 2005. Ultrafast $\mathrm{X}$-ray phase-enhanced microimaging for visualizing fuel injection process. SAE Technical Paper 2005-24-093. [doi:10.4271/2005-24-093]

Fitzgibbon, A., Pilu, M., Fisher, R.B., 1999. Direct least square fitting of ellipses. IEEE Transaction on Pattern Analysis and Machine Intelligence, 21(5):476-480. [doi:10.1109/ 34.765658]

Gao, Y., Deng, J., Li, C., et al., 2009. Experimental study of the spray characteristics of biodiesel based on inedible oil. Biotechnology Advances, 27(5):616-624. [doi:10.1016/j. biotechadv.2009.04.022]

Gao, Y., Huang, W., Gao, Y., et al., 2013. A study on the hole-to-hole spray variation based on nozzle internal structure. SAE Technical Paper 2013-01-1611. [doi:10. 4271/2013-01-1611]

Huang, W.D., Wu, Z.J., Gong, H.F., et al., 2013a. Effect of nozzle geometry on macroscopic behavior of diesel spray in the near-nozzle field. SAE Technical Paper 2013-011587. [doi:10.4271/2013-01-1587]

Huang, W.D., Gao, Y., Li, Z.L., et al., 2013b. Threedimensional investigations of flow characteristics in a diesel nozzle. Atomization and Sprays, 23(4):343-361. [doi:10.1615/AtomizSpr.2013006939] 
Kao, C.C., Shih, A.J., 2007. Form measurements of microholes. Measurement Science and Technology, 18(11): 3603. [doi:10.1088/0957-0233/18/11/045]

Kastengren, A.L., Tilocco, F.Z., Powell, C.F., et al., 2012. Engine combustion network (ECN): measurements of nozzle geometry and hydraulic behavior. Atomization and Sprays, 22(12):1011-1052. [doi:10.1615/AtomizSpr.2013 006309]

Macian, V., Bermudez, V., Payri, R., et al., 2003. New technique for determination of internal geometry of a diesel nozzle with the use of silicone methodology. Experimental Techniques, 27(2):39-43. [doi:10.1111/j.17471567.2003.tb00107.x]

Ohrn, T.R., Senser, D.W., Lefebvre, A.H., 1991. Geometrical effects on discharge coefficient for plain-orifice atomizers. Atomization and Sprays, 1(2):137-153. [doi:10.1615/ AtomizSpr.v1.i2.10]

Payri, R., Salvador, F.J., Gimeno, J., et al., 2008. Diesel nozzle geometry influence on spray liquid-phase fuel penetration in evaporative conditions. Fuel, 87(7):1165-1176. [doi:10. 1016/j.fuel.2007.05.058]

Peiner, E., Balke, M., Doering, L., 2009. Form measurements inside fuel injector nozzle spray holes. Microelectronic Engineering, 86(4-6):984-986. [doi:10.1016/j.mee.2008. 12.016]

Reitz, R.D., 1978. Atomization and Other Breakup Regimes of a Liquid Jet. PhD Thesis, Princeton University, Princeton, USA.

Wu, Z.J., Zhu, Z.Y., Huang, Z., 2006. An experimental study on the spray structure of oxygenated fuel using laserbased visualization and particle image velocimetry. Fuel, 85(10-11):1458-1464. [doi:10.1016/j.fuel.2005.12.024]
Wu, Z.J., Li, Z.L., Huang, W.D., et al., 2012. Comparisons of nozzle orifice processing methods using synchrotron Xray macro-tomography. Journal of Zhejiang UniversitySCIENCE A (Applied Physics \& Engineering), 13(3):182188. [doi:10.1631/jzus.A1100252]

\section{中文概要}

题 目: 喷油嘴喷孔直径和长度自动测量方法的开发和应 用

目 的：直喷内燃机工作过程中, 燃油通过喷油嘴上的微 小喷孔（直径为 100-200 $\mu \mathrm{m}$ ) 输送到燃烧室内进 行燃烧做功。为定量研究喷孔直径和长度等结构 参数对燃油输运过程的影响, 首先需要实现对这 些结构参数的高精度测量。

创新点：对喷油嘴喷孔入口和出口等重要结构特征进行精 确地定义, 实现喷油嘴微小喷孔的直径和长度的 高精度自动测量。

方 法：1. 基于同步辐射 $\mathrm{X}$ 射线 $\mathrm{CT}$ 扫描技术实现喷油嘴 顶部结构的三维重构; 2. 结合几何学原理, 从这 些离散的三维数字结构数据中提取宏观几何特 征参数。

结 论: 1. 对喷油嘴喷孔结构特征参数进行了精确地定 义； 2. 基于微米级精度建立了喷油嘴喷孔直径和 长度的自动测量方法, 实现喷孔沿其轴线从入口 到出口的直径分布的自动测量。

关键词: 柴油喷嘴; 喷孔直径; 喷孔长度; $X$ 射线 $\mathrm{CT}$ 扫描 